\title{
Calculation of Chemical Shift for Ti via EELS White-Line-Ratio Method
}

\author{
Lindsay R. Slick ${ }^{1}$, Yao-Jen Chang ${ }^{1}$ and Thomas Zega ${ }^{1,2}$ \\ 1. Lunar and Planetary Laboratory, The University of Arizona, Tucson, AZ, USA. \\ 2. Materials Science and Engineering, The University of Arizona, Tucson, AZ, USA.
}

$3 d$ transition metals occur in a wide range of mineral phases in primitive meteorites. Their oxidation states are directly affected by thermodynamic conditions, and so can provide constraints on the temperature and pressure under which their host phases formed or last equilibrated. Hibonite, thought to condense directy from the early solar nebula at high temperatures [1], can contain mixed valence $\mathrm{Ti}^{3+}$ and $\mathrm{Ti}^{4+}$. [2] determined that the existence of reduced $\mathrm{Ti}^{3+}$ in hibonite was likely the result of oxygen-vacancy formation under reducing conditions, and experimentally related these valences to oxygen fugacity $\left(f \mathrm{O}_{2}\right)$. $\mathrm{Ti}$ and $\mathrm{V}$ have been found in mixed valence in planetary pyroxenes [3] which were also associated with $f \mathrm{O}_{2}$. These measurements were of bulk material, and provided model-independent constraints on the thermal landscape of the early solar nebula. We hypothesize that atomic-scale measurements of the oxidation states of $\mathrm{Ti}$ and $\mathrm{V}$ could also provide new insights into the crystal chemistry and structure of planetary materials.

Electron energy-loss spectroscopy (EELS) has become a leading method in high spatial resolution and high energy resolution. EELS coupled to an aberration-corrected scanning transmission electron microscope (STEM) enables direct analysis of atomic columns [4-7]. There are primarily two EELS approaches to the quantification of oxidation state of $3 d$ metals. Direct quantification of oxidation states can be achieved by fitting the energy-loss near-edge structure (ELNES) with linear combinations of reference spectra [7-9]. Alternatively, L2,3 edges ('white lines') experience a chemical shift tied to oxidation state [10]. To quantify $\mathrm{Ti}^{3+} / \mathrm{Ti}^{4+}$, [11] applied a method developed by [12] whereby the ratio of oxidation states is directly proportional to the ratio of intensities of EELS white lines.

To establish the white-line-ratio method under our own calibrations, instrumentation, and optical conditions, we also acquired EEL spectra of Ti oxides of known $\mathrm{Ti}^{3+} / \mathrm{Ti}^{4+}$ content (Table 1). Spectra were acquired using a 200keV aberration-corrected Hitachi HF5000 S/TEM equipped with a Gatan Quantum ER electron energy-loss spectrometer. Spectra were acquired in TEM mode using a $2.5 \mathrm{~mm}$ entrance aperture and a dispersion of $0.01 \mathrm{eV} / \mathrm{ch}$. Spectra were processed using a Python script developed in house, which partially utilizes the hyperspy software package [13]. All spectra were corrected for channel-tochannel gain variation and dark current. Low-loss and high-loss spectra were calibrated via zero loss peak (ZLP) centering, an inverse power law $\left(\mathrm{AE}^{-1}\right)$ model was used to subtract background, and all spectra were independently normalized such that their highest intensities were set to 1 . The average energy resolution calculated by the full-width-half-maximum intensity of the ZLP over all spectra is approximately $0.26 \mathrm{eV}$. The white-line ratio method requires knowledge of the chemical shift of reference materials, and the edge onset (critical ionization energy, $E_{c}$ ) serves as a marker for such shift. The $E_{c}$ for our spectra were determined with a derivative approximation, such that the slope of the tangent line associated with edge onset was demonstrably positive when compared to local slope variation in the preceding noise (a minimum slope value of $9.1 \times 10^{-4}$ achieves these conditions for our data set).

Using this approach, we have calculated a shift in edge onset as a function of oxidation state over multiple sets of reference spectra. Figure 1 shows a representaive sample of spectra from our data set. Calculated 
shifts averaged between $0.10 \mathrm{eV}$ and $0.47 \mathrm{eV}$ among successive valencies from $\mathrm{Ti}^{2+}$ to $\mathrm{Ti}^{4+}$. Direct comparison to literature required calculating shifts with respect to the lowest-energy edge maxima (the second maxima in the case of $\mathrm{TiO}_{2}$ due to splitting), which can be seen in Table 1. These shifts, ranging from $0.17 \mathrm{eV}$ to $0.85 \mathrm{eV}$, are comparable to our $\mathrm{E}_{\mathrm{c}}$ calculations, but all shifts are smaller than previously reported by [11]. The shift between $\mathrm{Ti}_{2} \mathrm{O}_{3}$ and $\mathrm{Ti}_{3} \mathrm{O}_{5}$ specifically is consistently less than our energy resolution, and would appear to be statistically insignificant. Qualitative analysis of our ELNES dataset does not suggest contamination as a cause for a lack of significant shift. To increase statistics and decrease error, we will acquire more spectra for our working dataset. Analogous to [11], we will also calibrate spectra using additional ELNES other than the zero-loss peak, such as the amorphous carbon edge associated with the film on which our standards are prepared. This method should provide us with an additional check on spectral calibrations [14].

References:

[1] K Lodders, Ap. J. 591 (2003), p. 1220.

[2] JR Beckett et al., Geochem. Cosmochem. Acta 52 (1988), p.1479.

[3] SB Simon, SR Sutton and L Grossman. Geochem. Cosmochem. Acta 71 (2007), p.3098.

[4] PE Batson et al., Nature 418 (2002), p. 617.

[5] Varela et al., Phys. Review Lett. 92 (2004) \#095502.

[6] DA Muller et al., Science 319 (2008), p. 1073.

[7] Thompson et al., Meteoritics \& Planet. Sci. 51 (2016), p. 1082.

[8] Garvie and PR Buseck, Nature 396 (1998), p.667.

[9] TJ Zega, L Garvie and PR Buseck, American Min. 88 (2003), p.1169.

[10] R Brydson et al., in "Transmission Electron...in Materials Science", Eds. M.M Disko, et al.

(The Minerals, Metals, and Materials society, Warrendale, PA), p. 131.

[11] E Stoyanov et al., Amer. Mineralogist 92 (2007), p.557.

[12] PA Van Aken and B Liebscher, Phys Chem Minerals 29 (2002), p.188.

[13] F de la Pena et al., hyperspy/hyperspy v1.4.1 (2018).

[14] We gratefully acknowledge frunding from LARS grant \#80NSSC18K1475.

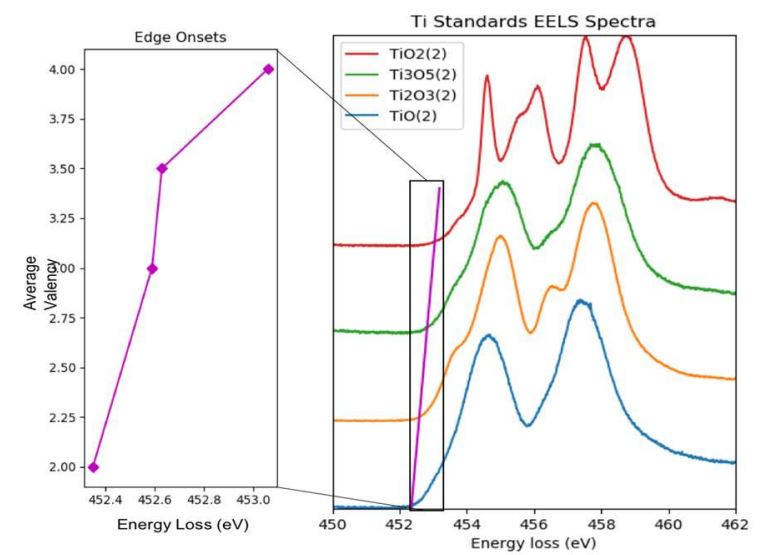

\begin{tabular}{|c|c|c|}
\hline Oxide & Average $\mathrm{L}_{3}$ peak & $\mathrm{L}_{3}$ (b) edge peak \\
\hline $\mathrm{TiO}$ & $454.48 \mathrm{eV}$ & $457.4 \mathrm{eV}$ \\
\hline $\mathrm{Ti}_{2} \mathrm{O}_{3}$ & $454.99 \mathrm{eV}$ & $458.9 \mathrm{eV}$ \\
\hline $\mathrm{Ti}_{3} \mathrm{O}_{5}$ & $455.16 \mathrm{eV}$ & $459.0 \mathrm{eV}$ \\
\hline $\mathrm{TiO}_{2}$ & $456.01 \mathrm{eV}$ & $460.0 \mathrm{eV}$ \\
\hline
\end{tabular}

Table 1. Energy loss of left-most $\left(\mathrm{L}_{3}\right)$ peak maxima for all Ti standards, listed from smallest to largest average valency. Right column indicates peaks found in table 2 of reference [11].

Figure 1. Ti Standards EEL spectra with edge onset indicators overlaid. Edge onset chemical shift is nearly linear with a mean average valency vs. energy loss slope of 0.34 . 